\title{
Expansión escolar privada e industrialización en España (1955-75). El caso de Miranda de Ebro
}

\section{Private school expansion and industrialisation in Spain (1955-75). The case of Miranda de Ebro}

\author{
M. ${ }^{a}$ Gloria Méndez VeGa ${ }^{1}$ \\ itxane@hotmail.com \\ Universidad Nacional de Educación a Distancia, UNED, España
}

\begin{abstract}
Resumen:
En el presente artículo se relaciona el crecimiento industrial desarrollado en España a partir de la llegada de los gobiernos tecnocráticos, en los últimos tres lustros del franquismo, con la explosión escolar, que se acusó de una manera especial en aquellas ciudades convertidas en polos industriales, el consiguiente déficit de puestos escolares en esta época y la respuesta privada a esta situación. Como paradigma, se estudia el incesante incremento de la demanda de estos puestos en una ciudad en expansión industrial, Miranda de Ebro (Burgos), y cómo esta demanda fue aprovechada por la iniciativa privada para plantear su oferta particular, en forma de grandes colegios de entidades religiosas o de pequeñas escuelas regentadas por particulares.
\end{abstract}

Palabras clave:

Expansión industrial; explosión escolar privada; tecnocracia; franquismo; escuelas privadas.

\begin{abstract}
:
This article connects the industrial development in Spain since the coming of the technocratic governments, during the last fifteen years of the Franco regime, with the boom of schooling, especially remarkable in new industrial towns, the consequent shortage of school vacancies during that time and the answer of the private school sector to the new situation. As a paradigm, this article studies the incessant increase of the demand of these vacancies in an industrial town, Miranda de Ebro (Burgos), and how this demand led private schools to start big religious schools or small secular schools.
\end{abstract}

\section{Key words:}

Industrial expansion; private school explosion; technocracy; Franco regime; private schools.

1 Dirección para correspondencia (correspondence address):

M. ${ }^{a}$ Gloria Méndez Vega. Fundación Cultural Profesor Cantera Burgos. Residencia

Fernán González. Camino de Anduva. Miranda de Ebro. 09200. Burgos (España). 
Expansión escolar privada e industrialización en España (1955-75). El caso de Miranda de Ebro

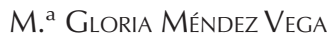

\section{Résumé:}

Cet article relie le développement industriel initié en Espagne à partir de l'arrivée des gouvernements technocratiques, pendant les trois derniers lustres du franquisme, avec l'explosion scolaire, accusée d'une manière particulière dans les nouvelles villes industrielles, le conséquent déficit de places scolaires pendant cette époque-là et la réponse privée à cette situation. Comme exemple, on étudie I'accroissement continu de la demande de ces places dans une ville en développement industriel, Miranda de Ebro (Burgos), et aussi comment par l'initiative privée a profitée de ce besoin pour offrir ses propre postes, sous forme de grands établissements religieux ou bien de petites écoles gérées par des particuliers.

Mots clés:

Développement industriel; explosion scolaire privée; technocratie; franquisme; écoles privées.

Fecha de recepción: 7-7-2015

Fecha de aceptación: 21-4-2016

\section{Introducción}

Partiendo del contexto nacional y local del momento (España/ Miranda de Ebro/1955-1975), se analizará la evolución de la respuesta privada ante el déficit de puestos educativos, así como la repercusión y duración de dicha respuesta, condicionada por la actuación a este respecto de la administración pública. Se comparará la situación con la que se describe en estudios nacionales (el Libro Blanco, 1969) e internacionales (Proyecto Regional Mediterráneo, 1963).

\section{El contexto nacional}

El final de la guerra civil trajo a España un régimen dictatorial que perduraría casi cuatro décadas. En 1955, inicio de este estudio, el país vive una situación económica angustiosa, a pesar de haber sido llevada a cabo en 1951 "la primera reforma gubernamental importante del nivel superior de la administración ministerial" (Crespo Montes, L. 2003. p. 31) y de haber sido España objeto de ayudas económicas entre 1953 y 1956. El malestar se manifiesta en forma de protestas obreras y revueltas estudiantiles. Franco se ve avocado a un nuevo cambio de gobierno, incluyendo esta vez ministros tecnócratas, muchos pertenecientes al Opus Dei. Desde 1957 a 1974 ocuparán los principales puestos de adminis- 
tración del estado, impulsando una política de crecimiento económico orientada a la exportación e integrando a España dentro del sistema capitalista mundial (Casanova, J. y Gil, C. 2009. pp. 267-268).

El déficit de reservas lleva a promulgar un Plan de Estabilización en 1959, que eliminará los obstáculos a las importaciones y conseguirá la concesión de préstamos por parte de organismos internacionales (FMI y OCDE). Además, a partir de 1963, son puestos en marcha tres planes de desarrollo: Primer Plan de Desarrollo (1964-1967), Segundo Plan de Desarrollo (1968-1971) y Tercer Plan de Desarrollo (1971-1975). Con ellos se apuesta por el sector industrial, haciendo más eficiente la estructura empresarial y creando polos de desarrollo. Desde 1959, industrias y servicios se convierten en las actividades fundamentales de la economía del país y aumenta la renta per cápita. Los autores difieren en la cuantía de este aumento: Carreras y Tafunell afirman que se multiplicó por 2.5 la renta por habitante (Carreras, A. y Tafunell, X. 2010. p. 345.); Pamela O'Malley habla de un 3.5\% en los años sesenta (O'Malley, P. 2010. p. 49) y Casanova y Gil, de un 7\% anual entre 1960-1973 (Casanova, J. y Gil, C. 2009. p. 269.). Con el incremento del poder adquisitivo, los españoles se lanzan al consumo de nuevos bienes (electrodomésticos, vehículos...).

Ahora bien, la modernización técnica de la empresa trajo como contrapartida una menor demanda de trabajadores no especializados, lo que provocó la emigración de alrededor de un millón de personas a países extranjeros (Francia, Alemania, Suiza...).

Hubo una emigración más trascendente a nivel social: la interior. La población agraria (especialmente la de Andalucía, las dos Castillas y Extremadura), atraída por el tipo de trabajo, vida y oportunidades que proporcionaba la gran ciudad, se dirigió a las urbes industrializadas del norte y levante español (Carreras, A. y Tafunell, X. 2010. p. 271).

Por otra parte, un fenómeno social iba a transformar la economía y los hábitos en esos años: el turismo internacional. España, por su clima, costas y nivel de vida, se convierte en el destino vacacional de muchos europeos. Sus divisas contribuirán al Ilamado milagro económico y a los cambios de comportamiento social.

A nivel político, las radicales transformaciones del país obligan a un intento de modernización institucional, que se traduce en leyes como la de Prensa (1966), la Ley Orgánica del Estado, La ley de Libertad religiosa y la Ley de Representación Familiar en 1967, la Ley de Educación de 
Expansión escolar privada e industrialización en España (1955-75). El caso de Miranda de Ebro

M. a Gloria Méndez VeGa

1970, de especial interés en nuestro objeto de estudio y analizada en diversas obras (Almeida, 1987; CNIDE, 1986; García Hoz, 1980; MEC, 1973; Navarro Sandalinas, 1990; Orden Hoz, 1985; Puelles Benitez, 1992 y Viñao, 1992) y la Ley Sindical de 1971. Mientras todo esto sucede, siguen vivos los movimientos contestatarios al régimen (políticos, sindicales, estudiantiles), reprimidos duramente por este: estado de excepción en 1969 y 1970, consejo de guerra en Burgos en 1970 contra 16 militantes de ETA...

La agonía del régimen se produce desde 1973, con el asesinato del vicepresidente del gobierno, Luis Carrero Blanco, a manos de ETA, y con la muerte del propio Jefe del Estado, el 20 de noviembre de 1975.

En cuanto a la situación de la educación en España, esta queda muy bien descrita en el Ilamado Libro Blanco², autoevaluación de la situación de la educación en España que se llevó a cabo como paso previo a la promulgación de la Ley General de Educación de 1970. En él se dice que durante el curso 1966-67 la cifra de niños en edad escolar obligatoria no escolarizados ascendía a más de medio millón, lo que suponía más del $12 \%$ de los niños de esa edad. Los principales motivos de esa situación, afirma, eran el desajuste entre la ampliación de la edad de escolaridad obligatoria y la previsión de puestos escolares para los niños que entraban en ese periodo de enseñanza, el crecimiento vegetativo de la población y el no cumplimiento de los objetivos a este respecto del Primer Plan de Desarrollo.

La creación de centros privados, con el fin de ofrecer puestos escolares, se había hecho sin una planificación que cuidase la conexión con la red pública. En el Libro Blanco se afirma que el criterio dominante para su apertura había sido su viabilidad económica, sus posibilidades de ser una actividad rentable. Estos colegios de pago establecían sus tarifas de manera libre, salvo en el caso de que fueran beneficiarios de subvención estatal, en cuyo caso la cuota mensual no podía exceder de la establecida para las Ilamadas permanencias en las escuelas públicas. (MEC, 1969. pp. 43-45)

La escasez secular de puestos escolares en nuestro país se sumaba al amplio crecimiento de la demanda de estos, creando un panorama de déficit de centros escolares al que el Estado debía dar respuesta. Este proyectó la creación de 25000 aulas para el quinquenio 1957-61. En el

2 Informe emitido por el MEC titulado La educación en España. Bases para una política educativa, editado en 1969 por su Secretaría General Técnica. 
I Plan de Desarrollo Económico y Social (1964-67) se incluyó un programa de construcción de más de catorce mil aulas, proyecto casi conseguido. (MEC, 1969. pp. 155-158)

Por su parte el Proyecto Regional Mediterráneo (MEC-OCDE, 1963), realizado a instancias de la OCDE, habla de esta situación. En él se recoge que la enseñanza privada había sido tradicionalmente en España muy importante ${ }^{3}$, especialmente en la enseñanza media. El informe recoge que en esos momentos alrededor de las tres cuartas partes de los alumnos de enseñanza primaria acudían a las escuelas del Estado y la mayor parte del resto, a las escuelas de la Iglesia. En la enseñanza media general, sin embargo, la situación era totalmente distinta: solo el $17 \%$ de los alumnos lo eran de centros oficiales; alrededor del $35 \%$ de los estudiantes de bachillerato general acudían a centros de la Iglesia reconocidos y un $18 \%$, a otros centros privados reconocidos; el resto eran alumnos libres. Respecto a la formación profesional, se dice en el informe que más del $60 \%$ de los alumnos estudiaban en centros no oficiales; de estos, el $65 \%$ se repartía casi por igual entre los centros sindicales y privados no religiosos, un $24 \%$ estaba matriculado en los centros de la Iglesia y un $11 \%$, en las universidades laborales, entidades educativas financiadas por las mutualidades laborales, dependientes del Ministerio de Trabajo. En cuanto al bachillerato laboral, se afirma que la mayor aportación correspondía al Estado, seguido de la Iglesia (MECOCDE, 1963. pp. 45-47).

\section{El contexto local: Miranda de Ebro}

Durante esos años, Miranda de Ebro (en el noreste de la provincia de Burgos, en la confluencia de esta con Álava y La Rioja) participa de la tónica general del país. La ciudad tiene 21156 habitantes en 1955 y es un nudo de comunicaciones de primer orden. En ella confluyen dos líneas ferroviarias (Madrid-Irún y Bilbao-Castejón) y es atravesada por la carretera N-I. El ferrocarril, Ilegado en 1864, había cambiado el semblante de Miranda, haciendo que pasara de villa agraria a ciudad industrial y polo de inmigración. ${ }^{4}$

3 De hecho, la Ley de 1945 consideraba el sector público como subsidiario de la actividad privada, siendo las familias y la Iglesia las adjudicatarias del derecho a educar.

4 En 1907, el rey Alfonso XIII concede a la villa el título de ciudad. 
Expansión escolar privada e industrialización en España (1955-75). El caso de Miranda de Ebro

M. a Gloria Méndez VeGa

En 1925 se había instalado la primera industria fuerte, la Azucarera Leopoldo, pero la expansión industrial vendría con FEFASA ${ }^{5}$, productora de fibra artificial, constituida en 1940 e inaugurada en 1952. En su inauguración el ministro de Industria, señor Planell, había señalado: "La fábrica proporciona al Estado una economía de divisas que puede valorarse en quince millones de dólares anuales" (Ortiz, C. 2001. p. 58). Atraídas por esta empresa vendrían otras más. Así, diez años después, en 1962, se inaugurará REPOSA ${ }^{6}$, industria de resinas poliésteres. Casi inmediatamente a esta inauguración, Burgos, la capital de la provincia, es creada Polo de Promoción Industrial, en el Primer Plan de Desarrollo (1964-1967).

En ese contexto, TALGO decide instalarse a cinco kilómetros de Miranda, en 1965. Cuatro años después se constituye en la ciudad la Sociedad Industrias Químicas Altamira. El último hito industrial tendrá lugar en 1971, cuando se inaugure una de las primeras centrales nucleares de España: Santa María de Garoña, situada a cuarenta kilómetros de Miranda. Por último, en 1972 se constituirá la empresa Montefibre Hispania. ${ }^{7}$

Alrededor de esas importantes empresas, surgirá toda una constelación de industrias secundarias (textiles, alimentación, construcción, piel, calzado, talleres...).

La instalación en la ciudad de tantas factorías convirtió a Miranda en punto demandante de mano de obra. En la ciudad no existía la suficiente, empleado el grueso de su población activa en el ferrocarril. La mano de obra vendría de la comarca natural de Miranda, de provincias limítrofes o cercanas e, incluso, de Andalucía.

El perfil social de la ciudad cambiaría con este nuevo aporte humano; también su demografía: entre 1955 y 1980 la ciudad aumenta su población en un $75 \%$. Es un porcentaje importante, pero no tanto si lo comparamos con otras ciudades de parecido perfil y población inicial, tales como Eibar, Ponferrada o Avilés, por ejemplo, que partiendo de entre 16000 y 21000 habitantes, vieron aumentar su población en un $120 \%$ las dos primeras y en un $400 \%$, la tercera, datos que dan cuenta

5 Esta factoría de Miranda de Ebro será objeto de estudio monográfico por parte de Santamaría y Asenjo en 1988. Llegó a tener 3000 empleados.

6 Contará con una plantilla de 117 personas.

7 En 1973 Industrias Químicas Altamira se fusiona con Acsa Española y funda Montefibre Hispania. La empresa Ilegará a tener 300 empleados. http://www.economiadigital.es/es/notices/2013/10/montefibre_en_concurso_con_deudas_de_30_millones_46092.php. Consultada el 20-10-2014. 
de la transformación de la sociedad española, que pasa de una economía agraria a otra industrial.

Este aumento poblacional, tanto de aporte foráneo como del propio aumento vegetativo, conllevaría nuevas necesidades ciudadanas, tales como urbanización de nuevas zonas y construcción de viviendas; también una creciente demanda de puestos escolares.

En resumen, las consecuencias de la industrialización de Miranda de Ebro fueron:

- inmigración

- crecimiento vegetativo, debido a que el aporte migratorio pertenecía a una franja de población en edad fértil y a que la conducta nupcial y de natalidad de los españoles en estos años fue optimista y explosiva

- demanda de infraestructuras urbanas

- crecimiento económico

- modernización de la ciudad y de la vida ciudadana

- necesidad continuada de incrementar la oferta de puestos escolares

A esta necesidad darían respuesta tanto la iniciativa pública como la privada. El presente artículo se ocupará en concreto de la inmediata respuesta dada por esta última: tanto la de las congregaciones religiosas como la de muchos particulares mirandeses, en condiciones de hacerlo, que vendrían a ocupar un nicho de mercado que la iniciativa pública tardaría en cubrir, envuelta en las dificultades económicas y administrativas propias de este tipo de acción estatal que consiste en construir y crear unidades escolares.

\section{Situación de partida: el déficit de puestos educativos en la ciudad}

En 1955 Miranda contaba con 27 centros educativos. En zona urbana, solo dos eran de carácter público en la enseñanza primaria: Escuelas Aquende y Escuelas de Allende, con una matrícula de unos cuatrocientos alumnos cada una ${ }^{8}$. La primera de ellas, abierta durante la II República, estaba situada en el casco histórico de la ciudad, (margen derecha

8 Aproximadamente, el 25\% de los alumnos escolarizados. 
Expansión escolar privada e industrialización en España (1955-75). El caso de Miranda de Ebro

M. a Gloria Méndez VeGa

del Ebro, río que divide la ciudad en dos zonas diferenciadas); Allende, inaugurada a principios de siglo, fue la primera Escuela de la Villa y estaba situada en la primera zona de ensanche urbano tras la desaparición de sus murallas medievales (margen izquierda). No obstante, públicas eran también las siete escuelas de los otros tantos barrios o pedanías de la municipalidad (Ayuelas, Bardauri, etc.). Otro centro más de carácter público era el instituto Fray Pedro de Urbina, que funcionaba en precario en unas instalaciones que no le eran propias (en la Escuela Aquende). Existía también una Escuela Taller de Formación Profesional, de la Obra Sindical.

Había, sin embargo, dieciséis centros de titularidad privada. De ellos, cuatro pertenecían a entidades religiosas: Sagrados Corazones, Sagrada Familia, Jesuitas e Hijas de San José; masculinos los dos primeros y femeninos los otros dos. El resto de los centros eran pequeñas escuelas propiedad de particulares, salvo la pequeña escuela parroquial San Nicolás y la escuela perteneciente a la empresa Azucarera Leopoldo. Excepto estos dos últimos, todos tenían como titulares a maestros que por propia iniciativa habían decidido impartir clases, dada la demanda que de puestos escolares había en esos momentos en la ciudad. Traducido a cifras porcentuales, estos datos serían: el $37.7 \%$ eran centros estatales; el $3.7 \%$ lo dirigía la obra Sindical (o sea, el $41.4 \%$ de los centros pertenecían a la Administración). El $58.6 \%$ eran centros de titularidad privada: el $18 \%$, religiosos, el $40.7 \%$ de particulares y el $3.7 \%$, de la empresa azucarera. Es decir, que la enseñanza era gratuita para los niños mirandeses que podían acceder a Aquende, Allende, la escuela de Azucarera, las de las pedanías o al instituto. El resto había de pagar si recibía instrucción.

En Miranda de Ebro el grave problema del déficit de puestos escolares queda registrado reiteradamente en las actas de los plenos del Ayuntamiento, las cuales plasman la preocupación del consistorio por el asunto y las diversas acciones que ejecuta este para intentar resolverla o paliarla. También las actas de los colegios públicos recogen el gran número de niños en edad escolar que se quedaba sin obtener plaza en ellos. Incluso la prensa local enfoca esta realidad. Así, con motivo del comienzo del curso 1956-57, los diarios vuelven una vez más a referirse a la escasez de puestos escolares afirmando que "si no fuera por la gran cantidad de colegios particulares y escuelas en todas las calles ¿quién solventaría el problema de la enseñanza en Miranda? Esta es la causa de 
que muchos abran por su cuenta una escuela particular, que a los pocos días se ve invadida de alumnos" (El Pensamiento Alavés, Vitoria. Hemeroteca Municipal de Vitoria. 1956. Vol. 3. 20-9-1956).

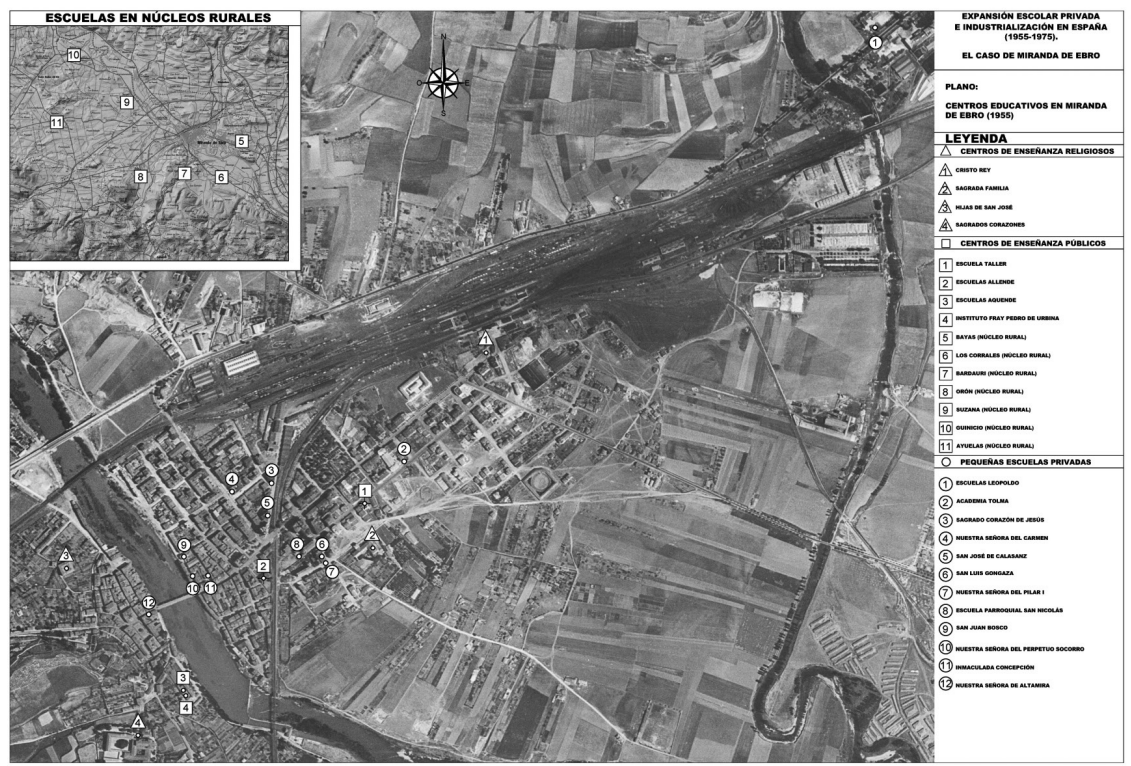

Mapa 1. Centros educativos en Miranda de Ebro en 1955.

Fuente: Elaboración propia. Fotografía: Instituto Tecnológico Agrario de la Junta de Castilla y León.

\section{La respuesta de la iniciativa privada ante la falta de puestos escolares}

Ante esta realidad, la iniciativa privada se mueve en la ciudad. Para empezar, entre 1956 y 1960 hay que hacer notar la creación de lo que serán dos grandes centros educativos de este carácter: un centro escolar en la barriada Ntra. Sra. de los Ángeles y el Seminario Marista.

La fundación del primero fue promovida por la empresa FEFASA. Dicha empresa había situado junto a su factoría un poblado para sus empleados, al cual dotó de servicios tales como economato y parroquia. En 1959 la empresa solicita a la administración educativa la autorización para el funcionamiento de una escuela, concedida unos años después. 
Expansión escolar privada e industrialización en España (1955-75). El caso de Miranda de Ebro

M. a Gloria Méndez VeGa

El colegio será denominado La Salle (FEFASA), porque son religiosos de esta congregación los que se ocuparán de la labor educativa. Contaba con tres clases para niños varones («BOE» núm. 76, de 29 de marzo de 1960, páginas 3997 a 3997. Sección: III. Otras disposiciones. Departamento: Ministerio de Educación Nacional).

El Seminario Marista, por su parte, se establecería en 1957 en lo que antes había sido un sanatorio, en el término de Fuentecaliente. Como seminario que era, funcionaba en régimen de internado para futuros novicios.

Además de estos grandes centros, en estos años tres escuelas particulares, regentadas por un único docente cada una, se sumarán a las ya existentes de este tipo. Son el centro San Juan Bosco, en calle Sorribas, cuya titular era Lorenza Martínez; Ramón y Cajal, regentado por Margarita Gómez y situado en la calle del mismo nombre, en el centro de la ciudad, y San Antonio, no lejos de la anterior, en calle Arenal, a cuyo frente está Félix Figuero.

En el quinquenio 1961-1965, el colegio de FEFASA se verá ampliado con dos unidades de párvulos, de ochenta plazas, que atenderán religiosas de Acción Parroquial (Archivo Histórico Provincial de Burgos. Sección Educación. Signatura 1069/2). De esa época es también la instalación de una nueva escuela particular, Ntra. Sra. del Pilar, cuya titular es Pilar López Uría. Como centro público, surge la escuela de la barriada Juan Yagüe, del mismo nombre.

En el lustro 1966-70 los dos primeros grandes colegios que se abren son religiosos, uno masculino y otro femenino. El primero de ellos es el Seminario Juan XXIII, que sería inaugurado en 1967. Previamente, en 1962, había Ilegado el hermano Buenaventura Meys, secretario de la Congregación de los Hermanos de la Inmaculada Concepción, fundada en Maastricht (por lo que el colegio fue conocido como "de los Holandeses"), con el fin de preparar lo necesario para el establecimiento de un primer grupo de cinco hermanos en pisos particulares. La construcción de una casa de formación, en los terrenos cercanos a la Barriada Yagüe, empezaría en 1964. Fue proyectado para recibir 230 alumnos internos, que realizarían en él los estudios de Bachillerato y Magisterio. Contaba el colegio con notables instalaciones deportivas (Arranz, J.1980. Pág. 273). El arquitecto, Rafael Gil Albarellos, daría al edificio un aspecto muy peculiar, casi fabril.

El segundo gran centro religioso se ubicará unos metros más al oeste 
de él, en Carretera de Orón, 98. Se trata del colegio de las Religiosas Franciscanas de Montpellier. Sus alumnas cursaban el Bachillerato Elemental como alumnas libres del Instituto de Miranda. Funcionó también como centro de Primaria, reconocido por OM de 30/1/1967.

En este periodo de 1965-70 también hay pequeñas escuelas privadas que se siguen abriendo. Hay tres que aparecen en 1966: Ntra. Señora de Covadonga, Virgen Niña y Ntra. Sra. de la Esperanza.

Ntra. Sra. de Covadonga la regenta Carmen Blanco y se halla situada cerca de otras de este tipo, en la calle Arenal, 10. Tuvo su creación definitiva en 1971 (primeramente la administración educativa daba autorizaciones provisionales). En el curso 1966-67 los alumnos pagaban 125 pesetas mensuales (Archivo Histórico Provincial de Burgos. Sección Educación. Signatura 1069/4).

La escuela Virgen Niña, por su parte, estaba situada en Plaza del Mercado, 19. Se trata de la única escuela privada de titularidad laica, junto con la pequeña escuela Ntra. Sra. de Altamira, que hemos encontrado ubicada en la zona del casco antiguo de la ciudad. Su creación definitiva fue el 7 de agosto de 1968.

La tercera de estas escuelas, Ntra. Sra. de la Esperanza, tuvo un carácter muy especial. Es la escuela creada para niños con deficiencias mentales, los cuales hasta el momento no habían tenido tratamiento específico: o bien acudían a los centros existentes o sus familias optaban por no escolarizarlos. El colegio estuvo situado en un principio en el barrio La Charca, pero avatares diversos fueron cambiando su ubicación. Creado oficialmente por OM de 29 de abril de 1967, su representante durante años fue Francisco Javier Vilar Bourbon.

Según testimonia en entrevista personal Ana Isabel Barceló, una de las monitoras de esta escuela, la iniciativa había partido de un grupo de padres de niños con problemas psíquicos, quienes se reunieron por primera vez en junio de 1965. Se constituyó una Junta Rectora que realizó las gestiones oportunas para la creación de una delegación de ASPANIAS ${ }^{9}$ en Miranda y se abrieron cuentas en las Cajas de Ahorros para que la ciudadanía pudiera ingresar en ellas sus donativos. En julio de 1966, el diario El Pensamiento Alavés (Vitoria 1966. Hemeroteca Municipal de

9 Aspanias es una asociación fundada en Burgos en 1964. Está formada por padres y familiares de personas con discapacidad intelectual y de desarrollo. Su objetivo, defender los derechos e igualdad de oportunidades de estas personas, trabajando por su integración social y laboral. http://www.aspaniasburgos.org/aniversario500/. Consultada 10-3-2015. 
Expansión escolar privada e industrialización en España (1955-75). El caso de Miranda de Ebro

M. a Gloria Méndez VeGa

Vitoria. 1966. Vol. 2. 20-7-1966), refiere que varios padres de los futuros alumnos habían comenzado los trabajos de habitación para la futura escuela. Los materiales constructivos fueron donados por industriales mirandeses. Según afirma, las autoridades, y el alcalde en particular, José María Aragüés, también se implicaron en esta obra. En septiembre de 1966 el colegio comenzaría su funcionamiento, con un total de once alumnos y con un profesor de Educación Especial.

Finalmente, en 1968 se abrirá una más de estas pequeñas escuelas privadas: Ntra. Sra. del Rosario, en la calle Alfonso VI, donde había otras más de este tipo. La titular es Rosario Alonso.

En el último lustro del franquismo, a pesar de la nueva norma educativa de 1970 y de lo avanzada que se encontraba en ese momento la creación de puestos escolares, tanto públicos como de grandes colegios religiosos, todavía hay particulares que deciden abrir establecimientos educativos. Es el caso de las escuelas Padre Damián y San Agustín, que abren en 1972, y de las de Virgen de Begoña y Centros Docentes Reunidos, que lo hacen en 1974.

Padre Damián, de dos unidades de preescolar, tenía su dirección en la calle Condado de Treviño, 72 bajo, zona alejada del centro. Su titular fue Julio Bañuelos (Archivo General de la Administración. Sección MEC. Legajo 55003. Expte. 6155). San Agustín, por su parte, estuvo en el número 10 de la calle del mismo nombre, en pleno centro urbano. Tuvo como titular a Segismunda Rámila e impartió niveles de preescolar y EGB. Antes de su creación hubo de sortear varias dificultades ya que en 1972 un informe de la Unidad Técnica (APB. Sec. EDU. Sign. 1069/14) describe la escuela (un tercer piso, 11 metros cuadrados) y dice que puede matricular 20 niños como máximo. Dos meses después, la inspectora Encarna Vergara emite informe negativo sobre el centro por sus malas condiciones y no autoriza su creación (APB. Sec. EDU. Sign. 1069/14). Suponemos que se solventarían parte de esas anomalías, ya que finalmente recibió la autorización provisional, impartiendo en el curso 197273 preescolar, $1^{\circ}, 2^{\circ}$ y $3^{\circ}$ de EGB. Se registra un precio por las clases de 200 pesetas mensuales. En 1979 el centro pidió ser clasificado como de EGB, pero no se admitió dicha solicitud.

La escuela Virgen de Begoña estuvo situada en la calle Santa Lucía, 56, bastante céntricamente. La fecha de su creación definitiva es la de 27 de octubre de 1976, pero funcionaba realmente con anterioridad, ya que el 9 de marzo de 1974, José Antonio Moneo Marina solicita la 
apertura del centro y adjunta plano del local, registro de penados, certificado médico y título de Magisterio. En 1973-74, su matrícula era de doce alumnos de cuatro años, dieciocho de cinco, tres de $1^{\circ}$ de EGB y cinco en $2^{\circ}$. El precio de las clases, 400 pesetas mensuales. Las profesoras eran en ese momento $M^{a}$ Blanca González Ortiz y Rosario Lomana Martínez, no su titular. El 22 de enero de 1975, la inspectora Encarna Vergara informa de la calificación definitiva del colegio como centro de Educación Preescolar, aunque admitía alumnos de los primeros cursos de EGB. En el expediente de ese centro encontramos un escrito por el que la Dirección General de Ordenación Educativa autoriza al cobro de 4.000 pesetas anuales por impartición de clases (Archivo Histórico Provincial de Burgos. Sección Educación Signatura 1070/4).

La escuela Centros Docentes Reunidos, sin embargo, fue un caso distinto en el grupo de pequeñas escuelas de titularidad privada laica. Parece ser que tras la Ley General de Educación de 1970 varios propietarios de algunas de estas pequeñas escuelas privadas existentes en Miranda de Ebro acometieron la estrategia empresarial de reunirse para formar un único centro, con el fin de poder hacer frente a las exigencias de la nueva ley respecto a condicionamientos pedagógicos. De hecho, un comunicado de la Unidad de Centros de 24 de enero de 1975, al señor director de Centros Docentes Reunidos considera clasificado en un solo centro (Centros Docentes Reunidos) los siguientes: Ntra. Sra. del Rosario, Luis Vives, Ntra. Sra. del Pilar, San Antonio, Ntra. Sra. de Covadonga, Padre Damián, Inmaculada Concepción y Ángel de la Guarda. El domicilio jurídico de Centros Reunidos será c/ Río Ebro, 1 (Archivo Histórico Provincial de Burgos. Sección Educación Signatura 1068/1). En su expediente aparece como propietaria Alicia Lapera. En el año 1974, la Administración indica a esta que aporte documentos para la consiguiente Clasificación y Transformación, requisito obligatorio para ser homologado como centro de EGB. En diciembre de ese año se desestima la Transformación, pero se le comunica que mientras haya necesidades de puestos escolares en Miranda se permitirá que funcione la Primera Etapa, asociado a un centro público.

La consecuencia más inmediata de la creación de Centros Docentes Reunidos es la desaparición de las ocho pequeñas escuelas privadas ya nombradas; ya en 1973 habían desaparecido otras seis: San José de Calasanz, Ntra. Sra. del Perpetuo Socorro, San Luis Gonzaga, Escuela Parroquial San Nicolás, Sagrado Corazón, y Ramón y Cajal. En 1975 
Expansión escolar privada e industrialización en España (1955-75). El caso de Miranda de Ebro

M. a Gloria Méndez VeGa

cerraría también Virgen Niña. Por su parte, los propios Centros Docentes Reunidos no sobrevivirían más que hasta 1978. También llegaría a su fin la escuela de la factoría Azucarera, que iría disminuyendo su matrícula, hasta cerrarse oficialmente en 1981.

Si a ello sumamos la desaparición del colegio de FEFASA, cuyo edificio cede la empresa a la administración educativa para asentar en él el Colegio Comarcal Ntra. Sra. de los Ángeles, podemos concluir que 1970-75 es el quinquenio del cierre de las escuelas privadas de titularidad laica, cuyo fin acaba consolidándose en el lustro siguiente.

El estudio detenido del conjunto de todas ellas nos ha hecho reparar en los siguientes aspectos que las definieron:

- ubicación: veintiuna de ellas estuvieron situadas en el barrio de Allende, en la parte más primitiva de este; es decir, muy centradas geográficamente. Más de ochocientos alumnos (el número de plazas en estas escuelas era de cuarenta alumnos) acudirían a estos establecimientos implantados en un radio espacial muy limitado. Solo dos centros estuvieron en la zona del Ensanche (una es la escuela para niños con deficiencias cognitivas), a unos diez minutos del centro, y dos son solo también las que escogieron situarse en el casco antiguo de la ciudad; ciertamente, en esta zona se hallaban ya dos grandes colegios religiosos (Hijas de San José y Sagrados Corazones), aunque las matrículas de estos colegios no se nutrieron principalmente de los niños censados en este barrio, y el colegio público Aquende. Por último, hubo tres escuelas cuyos titulares eran empresas (una de Azucarera Leopoldo y dos de Fefasa) y se hallaron situadas en la periferia, en las barriadas de viviendas de dichas factorías.

- tipo de local: el mayoritariamente escogido para situar estas escuelas fue una lonja a pie de calle, aunque también hubo algunas situadas en pisos de vecindad. Ninguno de estos locales contó con zona de recreo específica (salvo Centros Docentes Reunidos, al que se le obligó por la fecha de su creación) y, por supuesto, su dotación didáctica fue muy precaria en comparación con la de las escuelas públicas y los colegios privados religiosos.

- denominación: a pesar de ser escuelas de titularidad laica, los propietarios en su mayoría eligieron para sus escuelas nombres religiosos. De hecho, de las veintiocho escuelas, veintiuna llevaron nombres de santos o de advocaciones de la Virgen; una muestra 
de la impregnación de lo religioso en la vida cotidiana de la época. No obstante su nombre oficial, estas escuelas eran conocidas por el nombre propio del maestro o maestra: "la escuela de Doña Dolores", "la escuela de Don Antonio", "la escuela de Doña Matilde"

La implantación de la Ley General de 1970 exige a estas escuelas solicitar al MEC la consiguiente homologación y ajustarse a las condiciones que la legalidad impone. Unas lo hacen y otras optan por cerrar sus puertas; algunos de los titulares se jubilan, otros se incorporan como trabajadores de la enseñanza a colegios privados religiosos u optan por intentar acceder a la función pública. Un grupo de ellos emprende una iniciativa empresarial consistente en asociarse para formar un único centro (Centros Docentes Reunidos), de corta vida.

En resumen, la construcción de nuevos centros públicos permitió a las familias mirandesas acceder a puestos escolares gratuitos, con lo que el momento de existencia de estas pequeñas escuelas llegó a su fin, desapareciendo paulatinamente todas ellas.

A pesar de que el marco temporal de este estudio queda limitado a 1975, año de la muerte de Franco, consideramos de interés referir aquí cual fue el futuro inmediato de los grandes centros religiosos; de no hacerlo podría parecer que solo los pequeños particulares sufrieron las consecuencias de la dotación de suficientes plazas escolares públicas en la ciudad, algo que no concuerda con la realidad.

Desde 1975 y hasta los primeros años en democracia, Miranda de Ebro conocerá aún la inauguración de dos nuevos colegios públicos, pero también verá cerrar uno de los colegios religiosos de mayor potencia antaño: Hijas de San José. 
Expansión escolar privada e industrialización en España (1955-75). El caso de Miranda de Ebro

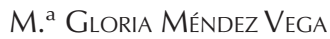

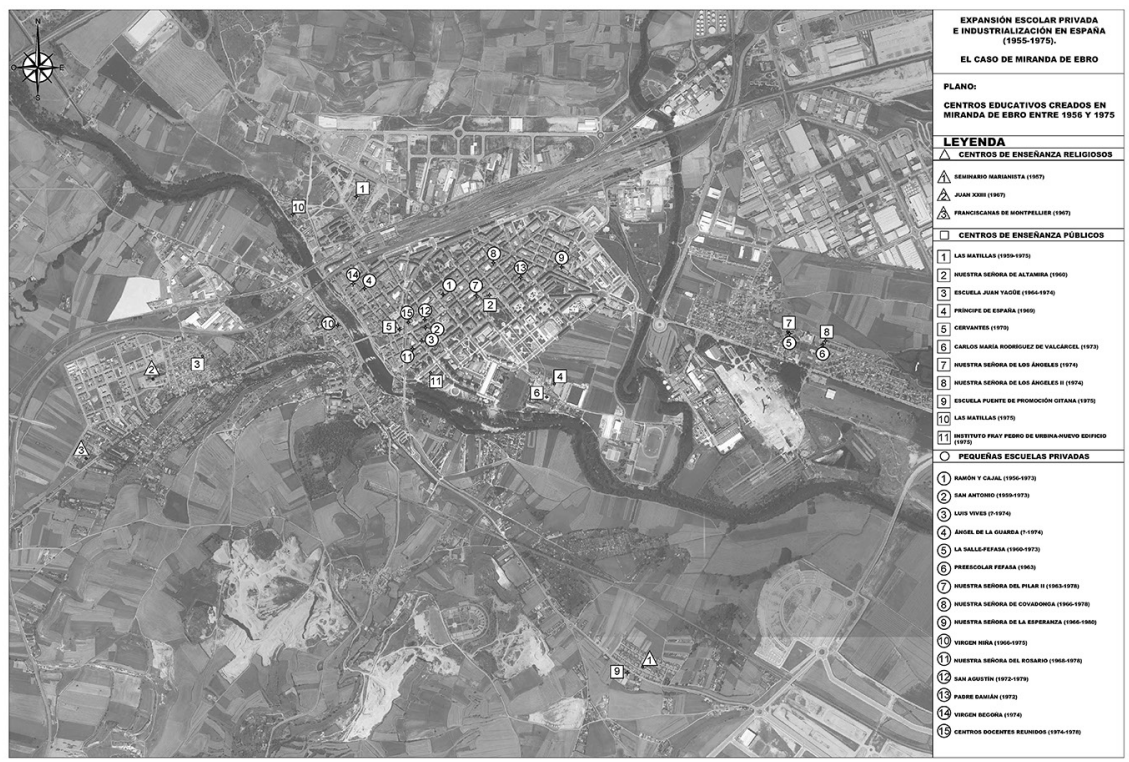

Mapa 2. Centros educativos creados en Miranda de Ebro entre 1956-1975. Fuente: Elaboración propia. Fotografía: Instituto Tecnológico Agrario de la Junta de Castilla y León.

Con la puesta en vigor de la Ley General de 1970, la comunidad religiosa titular de este centro optó por la Formación Profesional. Fueron extinguiendo los cursos del antiguo Bachillerato hasta su desaparición total en el curso 1975-76. Paralelamente, desde el curso 1972-73, el colegio impartía el Primer Grado de Auxiliar de Laboratorio y de Peluquería. Desde 1974-75, solo el Primer Grado de Auxiliar Administrativo. Las previsiones habían sido muy optimistas: se había pensado en 420 plazas de Formación Profesional para alumnas y, sin embargo, la respuesta fue tan alejada de las previsiones ${ }^{10}$ que el colegio cierra como tal en 1976 y la comunidad religiosa decide vender el edificio.

En un primer momento este es ofertado a la Administración educativa, pensando que puede interesarle contar con él para destinarlo a centro de EGB. Sin embargo, en 1978 un informe de la Inspección dice "estimo que no es procedente la adquisición del citado edificio, toda vez que con los colegios nacionales existentes y los que quedarán próximamente en funcionamiento construidos por el MEC quedan cubiertas las necesi-

10 El alumnado de Formación Profesional se quedaría en un 10\% de las expectativas. 
dades escolares de la población." (Informe de Inspección de 26-10-1978. Archivo Dirección Provincial de Educación de Burgos). La siguiente oferta se presenta al Ayuntamiento de Miranda de Ebro, quien finalmente adquirirá el edificio para destinarlo a Escuela Municipal de Música.

Así pues, en 1980 eran seis los centros religiosos que estaban en funcionamiento en la ciudad: Sagrados Corazones, Sagrada Familia, Juan XXIII, el Seminario Marista, Cristo Rey y Franciscanas de Montpellier. El colegio Juan XXIII, no obstante, cerrará solo tres años más tarde, en 1983. Su edificio será ocupado por los Padres Jesuitas para ubicar en él su centro, Cristo Rey, dado que el de su propiedad, en la calle La Estación, fue vendido y demolido para construir edificios de viviendas. En 1980 se impartían en este colegio estudios profesionales (en situación administrativa de adscripción al nuevo centro público de Formación Profesional recién inaugurado) de la rama del Metal, Delineante, Administrativo y Electricidad, además de párvulos y EGB. La reubicación del centro (en el extrarradio de la ciudad) y la construcción en la ciudad de dos nuevos colegios públicos en años posteriores, además del funcionamiento del público de Formación Profesional, harían que Cristo Rey fuera perdiendo gran número de matrícula.

Sin embargo, se hallaban a pleno rendimiento en ese momento Franciscanas de Montpellier y los dos únicos centros religiosos que aún persisten actualmente en la ciudad, los primeros en llegar a ella: Sagrados Corazones y Sagrada Familia. En el expediente del primero encontramos que ya en el año 1974 ambos habían sido objeto de subvención por parte del Estado (Archivo Histórico Provincial de Burgos. Sección Educación. Signatura 1269). 
Expansión escolar privada e industrialización en España (1955-75). El caso de Miranda de Ebro

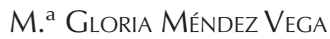

Tabla 1. Población, principales industrias, centros educativos y alumnado en Miranda de Ebro (1955-1975).

\begin{tabular}{|c|c|c|c|c|c|c|c|}
\hline Años & Población & $\begin{array}{l}\text { Industrias } \\
\text { (existentes y } \\
\text { creadas) }\end{array}$ & $\begin{array}{l}\text { Centros } \\
\text { Públicos }\end{array}$ & $\begin{array}{l}\text { Pequeñas } \\
\text { escuelas } \\
\text { laicas }\end{array}$ & $\begin{array}{l}\text { Grandes } \\
\text { colegios } \\
\text { religiosos }\end{array}$ & $\begin{array}{l}\text { Otros } \\
\text { centros }\end{array}$ & $\begin{array}{l}\text { Alumnos* } \\
\text { (Primaria }+ \\
\text { Secundaria) }\end{array}$ \\
\hline 1955 & 21156 & $\begin{array}{c}\text { Azucarera } \\
\text { FEFASA } \\
\text { Química } \\
\text { Quibasa } \\
+ \text { 159 PYMES }\end{array}$ & $10^{* *}$ & 10 & 4 & $3^{* * *}$ & 3200 \\
\hline $\begin{array}{l}1956 \\
1960 \\
\end{array}$ & $\begin{array}{l}21626 \\
27716 \\
\end{array}$ & REPOSA & +2 & +3 & +1 & $+1^{* * * *}$ & 4050 \\
\hline $\begin{array}{l}1961 \\
1965 \\
\end{array}$ & $\begin{array}{l}28027 \\
31419 \\
\end{array}$ & $\begin{array}{l}\text { INCOSA } \\
\text { TALGO }\end{array}$ & +1 & $+1 /-3$ & & $+1^{* * * * *}$ & 4300 \\
\hline $\begin{array}{l}1966 \\
1970\end{array}$ & $\begin{array}{l}31858 \\
34092\end{array}$ & $\begin{array}{l}\text { REDON } \\
\text { ALTAMIRA } \\
\text { C. NUCLEAR }\end{array}$ & +2 & $+4 /-1$ & +2 & & 4600 \\
\hline $\begin{array}{l}1971 \\
1975 \\
\end{array}$ & $\begin{array}{l}34535 \\
35603 \\
\end{array}$ & & $+4 /-8$ & $+4 /-9$ & & -2 & $\begin{array}{l}4821+832 \\
6826+632\end{array}$ \\
\hline
\end{tabular}

Fuente: Elaboración propia (datos: Padrón municipal, Archivo Histórico Municipal, Archivo Histórico Provincial, Archivo Dirección Provincial de Educación)

Leyenda: (+) centros creados; (-) centros suprimidos

* Valores estimados; último quinquenio, valor real

** Tres en el casco urbano y el resto en las pedanías.

***Escuela Parroquial San Nicolás / Escuela Taller de la Obra Sindical /Escuela Azucarera Leopoldo

**** Escuela FEFASA masculina

$* * * * *$ Escuela FEFASA femenina

\section{Conclusiones}

A través de lo que ha desvelado el análisis de la documentación municipal, dotar de plazas escolares de carácter público a Miranda durante los años estudiados fue un proceso largo y complicado. Ello se debió a factores tales como la necesidad de dotación económica, el proceso burocrático legal y la lógica duración de la ejecución de las obras de construcción de nuevos edificios. Además, el aumento de población fue "pisando los talones" a la administración, que no termina de cerrar un frente cuando se le abre otro; es decir, construye centros, pero aún se necesitan más, ya que la población escolar sigue creciendo. No obstante, el tiempo de este estudio empieza con un grave déficit de puestos edu- 
cativos y acaba con una respuesta pública suficiente para las enseñanzas obligatorias.

En cuanto a los centros privados de titularidad religiosa, concluimos que en estos siete grandes centros (excluida la pequeña Escuela Parroquial San Nicolás) existen dos grupos distintos, con casi idénticos comportamientos. Por un lado estarían Cristo Rey, Hijas de San José, Juan XXIII, Franciscanas de Montpellier y Hermanos Maristas (aunque este es un caso singular, ya que el centro solo estaba destinado a futuros frailes de esta comunidad religiosa), los cuales tienen en común haber llegado a la ciudad a finales de los años cuarenta o en los cincuenta, construir unos edificios ambiciosos en cuanto a tamaño y encontrar rápida y positiva respuesta en Miranda a su oferta educativa. Sus matrículas son altas, viven momentos álgidos y son grandes protagonistas de la educación. Sin embargo, con el nuevo sistema educativo, la construcción de edificios de escuelas públicas y a veces por elecciones no acertadas (reubicación, opción por unas u otras enseñanzas) ven declinar sus matrículas, hasta el punto de que las respectivas comunidades toman la decisión de cerrarlos. En el otro grupo estarían los colegios Sagrados Corazones y Sagrada Familia, de gran antigüedad en la ciudad ambos, que albergaron como alumnos durante décadas, entre otros, a los hijos de las familias mirandesas más acomodadas. Su prestigio siguió perdurando y la gestión de sus respectivas administraciones tuvo mayor acierto o previsión de futuro, de tal manera que con el paso del tiempo siguieron manteniendo sus niveles de matrícula.

Cuando la red pública, en definitiva, fue corrigiendo el déficit de puestos escolares al que antes había dado respuesta la iniciativa privada, ya no había sitio para todos los centros educativos religiosos. Se sostuvieron los más fuertes o bien aquellos cuyas comunidades priorizaron la labor educativa en sus órdenes sobre otras labores pastorales.

En cuanto a las pequeñas escuelas de iniciativa privada, igual que en el caso de los colegios privados religiosos, es el déficit de puestos escolares lo que mueve a particulares a emprender el negocio de la enseñanza en la ciudad. Hasta un total de veintiocho pequeñas escuelas de titularidad privada laica llegaron a funcionar en los años estudiados. Y es que, a pesar de la capacidad para una matrícula numerosa que tenían los grandes colegios religiosos, todavía había hueco para estas escuelas menores, que llegarían a acoger entre todas unos mil alumnos (el equivalente a unos dos colegios privados religiosos). 
Expansión escolar privada e industrialización en España (1955-75). El caso de Miranda de Ebro

M. ${ }^{a}$ Gloria Méndez Vega

La implantación de la Ley General de 1970 exige a estas escuelas solicitar la consiguiente homologación al MEC y ajustarse a las condiciones que la legalidad impone. Unas lo hacen y otras optan por cerrar sus puertas.

Podemos concluir, pues, que la ciudad de Miranda de Ebro conoce su expansión industrial y su explosión escolar, y el consiguiente déficit de puestos escolares, al mismo tiempo que estos fenómenos tuvieron lugar en España. Su estratégica ubicación nacional y su dotación de infraestructuras, así como el hecho de hallarse en el ámbito de uno de los polos de desarrollo marcados por el gobierno, hicieron de esta localidad punto escogido para instalar importantes industrias y diversidad de ellas. Ello trajo consigo gran aporte de población foránea, lo que se traduciría en un notable crecimiento demográfico y en la consiguiente demanda de puestos educativos, tanto de educación primaria, como secundaria y profesional. En aplicación de las nuevas políticas educativas, las administraciones del ramo irán dando respuesta a esas demandas construyendo centros. Mientras esto se materializa, la iniciativa privada, seguramente de manera similar a cómo respondió en las zonas del país que vivían la misma situación, dará cabida a esta demanda hasta que la oferta de la pública sea suficiente, hecho que viene a producirse alrededor de 1980.

\section{Referencias}

Almeida Nesi, J. (1987). La escuela española un siglo después de Joaquín Costa en Revista de Educación, 283, 187-200.

Arranz, J. (1980). Miranda de Ebro hoy. Miranda de Ebro: Lifer.

Carreras, A., y Tafunell, X. (2010). Historia económica de la España contemporánea. Barcelona: Crítica.

Casanova, J. y Gil, C. (2009). Historia de España en el siglo XX. Madrid: Ariel.

Centro Nacional de Investigacion y Documentacion Educativa (1986). Examen de la política educativa española por la OCDE. Madrid: MEC.

Crespo, L. (2003). Mitos y ritos de la Administración española. Madrid: INAP.

Garcia Hoz, V. (1980). La educación en la España del siglo XX. Madrid: Rialp.

MEC. (1969). La educación en España. Bases para una política educativa. Madrid: Secretaría General Técnica.

MEC-OCDE. (1963). Proyecto Regional Mediterráneo. Las necesidades de educación y el Desarrollo Económico-Social de España. Madrid: MEC.

MEC. (1963). Ley General de Educación y Disposiciones complementarias. Madrid: MEC. 
Expansión escolar privada e industrialización en España (1955-75). El caso de Miranda

de Ebro

M. ${ }^{a}$ Gloria MÉndeZ VeGA

Navarro, R. (1990). La enseñanza primaria durante el franquismo (1936-1975). Barcelona: PPU.

O'Malley, P. (2010). La educación en la España de Franco. Madrid: Dens (Coord. Ed. Ruiz Reig, J.).

Orden Hoz, A. de la. (1985). Innovación pedagógica en la Reforma educativa de 1970. Madrid: Ruiz Berrio, J. Ed.

Ortiz García, C. (2001). 100 años de Miranda. Miranda de Ebro: Miranda.

Puelles Benitez, M. de. (1992) Tecnocracia y política en la reforma educativa de 1970, en Revista de Educación, N extraordinario, La ley de Educación 20 años después, 13-29.

Santamaría, M. y Asenjo, E. (1988). Fefasa:(1940-1972. Un gran complejo industrial en Miranda de Ebro. (Análisis económico y social de una empresa durante el franquismo. Miranda de Ebro: Instituto Municipal de la Historia.

Viñao Frago, A. (1992). La Educación General Básica. Entre la realidad y el mito. Revista de Educación. № extraordinario, La ley de Educación 20 años después, 47-71. 
